\title{
Molecular Transport across Lipid Membranes Controls Cell-Free Expression Level and Dynamics
}

\author{
Patrick M. Caveney ${ }^{1,2}$, Rosemary M. Dabbs ${ }^{2}$, William T. McClintic ${ }^{1,2}$, C. Patrick Collier ${ }^{1}$, Michael \\ L. Simpson ${ }^{*}, 1,2$ \\ ${ }^{1}$ Center for Nanophase Materials Sciences, Oak Ridge National Laboratory, Oak Ridge, TN, \\ 37831, USA \\ ${ }^{2}$ Bredesen Center for Interdisciplinary Research and Graduate Education, University of \\ Tennessee Knoxville/Oak Ridge National Laboratory, Knoxville, TN, 37996, USA \\ *Corresponding author: SimpsonML1@ornl.gov
}

\begin{abstract}
Summary
Essential steps toward synthetic cell-like systems require controlled transport of molecular species across the boundary between encapsulated expression and the external environment. When molecular species (e.g. small ions, amino acids) required for expression (i.e. expression resources) may cross this boundary, this transport process plays an important role in gene expression dynamics and expression variability. Here we show how the location (encapsulated or external) of the expression resources controls the level and the dynamics of cell-free protein expression confined in permeable lipid vesicles. Regardless of the concentration of encapsulated resources, external resources were essential for protein production. Compared to resource poor external environments, plentiful external resources increased expression by $\sim 7-$ fold, and rescued expression when internal resources were lacking. Intriguingly, the location of resources and the membrane transport properties dictated expression dynamics in a manner well predicted by a simple transport-expression model. These results suggest membrane engineering as a means for spatio-temporal control of gene expression in cell-free synthetic biology applications and demonstrate a flexible experimental platform to understand the interplay between membrane transport and expression in cellular systems.
\end{abstract}

\section{Keywords}

Cell-free, lipid vesicles, synthetic biology, membrane permeability

\section{Introduction}

Confined cell-free gene expression systems (Karim and Jewett, 2016; Moore et al., 2018; Pardee et al., 2016; Shin and Noireaux, 2012; Siegal-Gaskins et al., 2014; Siuti et al., 2011) are making strides (Perez et al., 2016) toward cell-like capabilities (Scott et al., 2016; Trifonov, 2011). Recent reports demonstrate important steps along this path, including genome replication (Sakatani et al., 2015), metabolism (Garcia et al., 2018), adaptation (Yoshiyama et al., 2018), and growth (Exterkate et al., 2017). However, little work has been reported on one of 
the key next steps - controlled molecular interactions with the external environment (Collier and Simpson, 2011).

This communication across the membrane is essential for highly complex cellular functions such as chemotaxis (Van Haastert and Devreotes, 2004), symbiosis (Braga et al., 2016), and collective action (e.g. biofilm formation (Flemming et al., 2016)). For cell-like systems, essential next steps like energy harvesting, require the controlled trafficking of molecules across the boundary between cell-like system and the external world. Recent work demonstrates the use of pore-forming proteins (Chalmeau et al., 2011; Noireaux and Libchaber, 2004) or optical treatment of vesicles (Caveney et al., 2019) to engineer transport across lipid membranes. When these transport processes involve molecules that support protein synthesis (e.g. ions, nucleotides, amino acids), transport and gene expression dynamics become entwined (Figure 1A).

Gene expression in cell-like systems is a function of both encapsulated and external expression resources (Figure 1B). Recently, we demonstrated an optical treatment protocol to make vesicle membranes uniformly permeable to molecules relevant to protein expression (Caveney et al., 2019). This technique reduced vesicle-to-vesicle variability in protein expression by making membrane transport properties uniform and by reducing the effects of stochastic seeding of the encapsulated resources. However, permeable membranes change the dynamics of gene expression by accentuating the role of molecular transport across the membrane. Here we show how the location (encapsulated or external) of the expression resources controlled the level and the dynamics of cell-free protein expression confined in permeable lipid vesicles. Regardless of the concentration of encapsulated resources, external resources were essential for protein production, increasing expression levels by $\sim 7$-fold. By sourcing essential molecular species, resource-rich external environments rescued expression when internal resources were lacking. Intriguingly, the location of resources and the membrane transport properties dictated expression dynamics in a manner well predicted by a simple transport-expression model. Since the membrane transport properties may be controlled in space and time using a simple optical treatment (Caveney et al., 2019), these results demonstrate the means for predictive spatiotemporal control of gene expression in cell-free synthetic biology applications. Furthermore, the experiments described here show a flexible experimental platform to understand the interplay between membrane transport and expression in individual cells or in groups of cells working cooperatively through cell-to-cell molecular signaling.

\section{Results and Discussion}

To study the dynamics of protein expression in permeable lipid vesicles, we tracked cell-free expression of Yellow Fluorescent Protein (YFP) confined in optically-permeabilized (Caveney et al., 2019) vesicles (Figure 2A and 2B; Methods). Vesicles were created by the emulsion-transfer method described previously (Caveney et al., 2016; Nishimura et al., 2014a; Noireaux and Libchaber, 2004). Briefly, an inner solution composed of the PURE system (Shimizu et al., 2001), a YFP encoding plasmid, pEToppY, and a fluorescent volume marker, AF647 conjugated to transferrin, were vortexed in paraffin oil containing 1-palmitoyl-2-oleoyl-glycero-3phosphocholine (POPC) to create reverse micelles. The reverse micelles were centrifuged 
through an oil-water interface into the outer solution to create vesicles (Figure 2A). Vesicles were imaged with z-stacks of 20 slices every 3 minutes for a 3-hour period using a Zeiss, LSM 710 confocal laser scanning microscope. This imaging protocol, light_max, permeabilizes the lipid membranes, allowing the transport of molecular species (e.g. nucleotides, amino acids) essential for expression (Caveney et al., 2019). The images of the AF647 at each time point (Figure 2B, Top) were used to locate individual vesicles possessing distinct boundaries with minimal overlap with neighboring vesicles. The dynamics of protein expression were inferred from the time histories (Figure 2B, Bottom Inset) of the measured YFP fluorescence (Figure 2B, Bottom) from these ROls.

We performed experiments using protocols defined by the concentrations (high $(\mathrm{H})$ or low $(\mathrm{L})$; Methods) of resources encapsulated within vesicles and those in the outer solution (Figure 2C). This led to four types of experiments: (1) Low encapsulated/low outer (LL); (2) High encapsulated/low outer (HL); (3) Low encapsulated/high outer (LH); and (4) High encapsulated/high outer $(\mathrm{HH})$. After 2 hours, when protein expression had stopped, the YFP fluorescence resulting from the LL protocol was nearly indistinguishable from background, indicating very low gene expression activity (Figure 3A). Conversely, three of these protocols $(\mathrm{HL}, \mathrm{LH}$, and $\mathrm{HH}$ ) led to significant protein expression activity as indicated by YFP fluorescence levels measurably above background (Figure 3B-D). These results indicate that if the expression resources were available, either encapsulated or within the outer solution, gene expression occurred. Yet, the location of the resources controlled both the level (Figure 3E) and the dynamics (Figure 3F) of expression.

With the benefit of a full complement of both encapsulated and external resources, the $\mathrm{HH}$ protocol led to the highest level of gene expression (Figure 3D). In agreement with a previous report (Carrara et al., 2018), high resource concentrations were essential to maximizing protein production as expression in the $\mathrm{HH}$ condition was nearly $\sim 7$-fold greater than either of the other two productive protocols. Intriguingly, $\mathrm{HL}$ and $\mathrm{LH}$ protocols produced similar levels of total protein at the 2-hour mark (Figure 3B, C, and E), yet displayed very different expression dynamics (Figure 3F). Regardless of the outer solution resource concentration, a high concentration of encapsulated resources led to a rapid onset of expression (Figure 3F). Conversely, expression in the low encapsulated resource environment was rescued by a high concentration of external resources, but only after a considerable delay (Figure 3F).

To better understand the dynamics of cell-free gene expression in permeable vesicles, we constructed a model that accounted for molecular transport across the membrane (Figure 4A). This model included three types of molecular species important in the expression process: (Q) encapsulated molecules (e.g. ribosomes, RNAP) that do not cross the membrane (immobile); (L) highly transportable species (e.g. small ions) that were both encapsulated and in the outer solution that readily crossed the membrane (highly mobile); and (R) slowly transported species (e.g. amino acids, nucleotides) that were both encapsulated and in the outer solution (lowly mobile). In the model, the internal concentration of these three species and the known decay in expression capacity with time (Caveney et al., 2016; Sun et al., 2013) controlled the rate of gene expression. The time dependence of expression capacity was modeled as an exponential 
decay (rate constant $k_{p}=0.00001^{*} e^{-0.0125^{*} t}$ ). The internal concentration of immobile species was set by the initial conditions (either high or low) and remained constant throughout the simulation (SI Figure 1D). The internal concentrations of the two mobile species (L) and (R) reached equilibrium with the external concentration of these species after a delay time set by the transport rates (SI Figure 1A-C). Since the highly mobile species were expected to equilibrate with the outer solution very quickly, this rate constant was set high $\left(\mathrm{k}_{\mathrm{Lin} / \mathrm{out}}=0.1^{*} \mathrm{t}\right)$. The lowly mobile rate constant was found by fitting to the transport transient of fluorescein into vesicles from the outer solution (Methods, SI Figure 2D). This resulted in time-variant transport process described by $k_{\text {Rin/out }}=0.00019^{*} t$, where transport across the membrane for the lowly mobile species increased with increased light exposure.

Gillespie simulations (Gillespie, 1977) of expression were run using the model for four conditions approximating the experimental $\mathrm{HH}, \mathrm{HL}, \mathrm{LH}$, and LL protocols, and simulation results reproduced the major experimental behaviors. Similar to experiments, the $\mathrm{HH}$ simulations resulted in the most protein. $\mathrm{HL}$ and $\mathrm{LH}$ simulations resulted in $\sim 3.4 \mathrm{x}$ and $14 \mathrm{x}$ less protein than $\mathrm{HH}$ (Figure 4B). Further, the $\mathrm{HH}$ and $\mathrm{HL}$ simulations (most encapsulated resources), had a rapid initial rise in protein production while the LH model has a significant time lag (Figure 4C) caused by the delayed arrival of the lowly mobile species. The variation in the amounts of protein made in the different modeling conditions was caused by the interplay between the time that resources arrived in the vesicle and the exponential decay in protein expression capacity.

Perhaps cell and cell-free synthetic biology differ most in the emphasis each place either on gene circuits or on the environment in which gene circuits reside. Cell-free synthetic biology is placing ever greater emphasis on controlling the gene circuit environment by manipulating confinement volume (Caveney et al., 2016), degree of macromolecular crowding (Norred et al., 2018), and the composition of cell extract (Garcia et al., 2018). A defining feature of the gene circuit environment in cells is the controlled transport of molecules across membranes. Such interplay between membrane transport and gene expression in cells play important roles in expression variability (Hansen et al., 2018a) and complex functionality like probabilistic fate determination (Hansen et al., 2018b). Furthermore, cells use membrane transport to take in energy molecules, sense the environment (Van Haastert and Devreotes, 2004), coordinate population behavior (e.g. quorum sensing (Miller and Bassler, 2001)), and share genetic material (e.g. horizontal gene transfer (Gogarten and Townsend, 2005)). It is an intriguing possibility that membrane engineering for controlled molecular transport between encapsulated and external cell-free expression environments may enable essential next steps toward synthetic systems that approach cell-like functional complexity.

\section{Vesicle Preparation}

\section{Methods}

Vesicles were made using the oil-in-water emulsion technique (Noireaux and Libchaber, 2004; Pautot et al., 2003) (Figure 2A). This method encapsulated a protein expressing inner solution in vesicles separated from an osmotically balanced outer solution. The inner solution was prepared using $10 \mu \mathrm{L}$ Solution A and $7.5 \mu \mathrm{L}$ Solution B of the PURExpress In Vitro Protein 
Synthesis Kit from New England Biolabs; $5 \mu \mathrm{L}$ of sucrose solution (1 M); $0.25 \mu \mathrm{L}$ of TransferrinAlexaFluor $647 ; 0.125 \mu \mathrm{L}$ of RNAsin $(40 \mathrm{U} / \mu \mathrm{L}) ; 0.418 \mu \mathrm{L}(1.67 \mathrm{nM})$ of YFP encoding pEToppY plasmid (Nishimura et al., 2014b) (200 ng; $478.2 \mathrm{ng} / \mu \mathrm{L}$ stock); and nuclease-free water to bring the total volume of solution to $30 \mu \mathrm{L}$. The inner solution was vortexed in $330 \mu \mathrm{L}$ of paraffin oil containing $30 \mathrm{mg}$ of POPC for 60 seconds. The resulting emulsion was layered above the outer solution and centrifuged at $13,000 \mathrm{~g}$ for 20 minutes at room temperature. The low concentration inner solutions were made diluting Solution A and Solution B with nuclease-free water to $1 / 3$ their standard concentrations.

\section{Outer Solution Preparation}

The outer solution for vesicles was mixed from frozen stocks before each experiment. $1.5 \mu \mathrm{L}$ Amino acid solution, $11.3 \mu \mathrm{L}$ of ATP $(100 \mathrm{mM}), 7.5 \mu \mathrm{L}$ of GTP $(100 \mathrm{mM}), 0.75 \mu \mathrm{L}$ of CTP (500 $\mathrm{mM}), 0.75 \mu \mathrm{L}$ of UTP $(500 \mathrm{mM}), 1.8 \mu \mathrm{L}$ of spermidine $(250 \mathrm{mM}), 3.75 \mu \mathrm{L}$ of creatine phosphate $(1 \mathrm{M}), 4.5 \mu \mathrm{L}$ of Dithiothreitol $(100 \mathrm{mM}), 0.75 \mu \mathrm{L}$ of Folinic Acid $(0.5 \mathrm{M}), 24 \mu \mathrm{L}$ of potassium glutamate $(3.5 \mathrm{M}), 11.3 \mu \mathrm{L}$ of magnesium acetate $(0.5 \mathrm{M}), 30 \mu \mathrm{L}$ of HEPES $(1 \mathrm{M}), 60 \mu \mathrm{L}$ of glucose $(1 \mathrm{M})$, and $141.8 \mu \mathrm{L}$ of autoclaved type I pure water for a total volume of $300 \mu \mathrm{L}$. The low concentration outer solutions were made by diluting the entire outer solution to $1 / 3$ its standard concentration.

\section{Vesicle Imaging}

Fluorescent images were obtained using a confocal microscope to track YFP expression and AF647 fluorescence for three hours (Figure 2B and 2B inset). The pellet of vesicles was collected with $100 \mu \mathrm{L}$ of the outer solution and pipetted onto a no. 1.5 glass bottom petri dish. The lid was placed on the petri dish to minimize airflow and evaporation of the $100 \mu \mathrm{L}$ outer solution and vesicle drop. Vesicles were imaged with the light_max protocol (Caveney et al., 2019). The petri dish was placed on a Zeiss LSM710 confocal scanning microscope with an incubation chamber warmed to $37^{\circ} \mathrm{C}$ and imaged every 3 minutes in a z-stack with a $20 \mathrm{x}$ air objective. Vesicles were imaged with three lasers: a $405 \mathrm{~nm}, 6.5 \mathrm{~mW}$ laser; YFP was excited with a $488 \mathrm{~nm}, 6.1 \mathrm{~mW}$ laser and fluorescent emission was collected from 515-584 nm; and AF647 was excited with a $633 \mathrm{~nm}, 1.67 \mathrm{~mW}$ laser and fluorescent emission was collected from 638-756 nm. Z-stacks were made of $\sim 21$ slices at $1 \mu \mathrm{m}$ intervals, and the aperture for each slice was 1.00 Airy Units (open enough to allow $\sim 1.5 \mu \mathrm{m}$ depth of light). The time the vesicles sat on the microscope before imaging was minimized (less than 15 minutes), allowing for imaging for most of the duration of protein expression.

\section{Data Acquisition and Analysis}

Average fluorescent intensities were measured with the FIJI TrackMate (Tinevez et al., 2017) (v3.8.0) plugin. TrackMate found spots with an estimated blob diameter of $10 \mu \mathrm{m}$ using the Laplacian of Gaussian detector. Spots that were found with an estimated diameter $<5 \mu \mathrm{m},>19$ $\mu \mathrm{m}$, or contrast $<0$ were removed from the data set. We used the simple Linear Assignment Problem (LAP) tracker to link spots across z-stacks in time to create traces. Traces that had missing frames, traveled $>5 \mu \mathrm{m}$ between frames, or tracked for $<45$ of the 60 frames were removed from the data set. Protein concentration (in arbitrary units) was measured as the average fluorescent intensity for each vesicle at each time point. 


\section{Determining Vesicles Indistinguishable from Background}

Background ROls were determined from the AF647, volume marker, channel. Intensity of these ROls was measured in the YFP channel. A sample of vesicles with clearly defined edges by eye in the AF647 channel but no clear edges by eye in the YFP channel were measured for their YFP intensity. This value, $0.6147 \mathrm{AU}$, was slightly above the background value measured and was used as a cut-off for vesicles that were indistinguishable from background fluorescence.

\section{Measuring the Transient of Membrane Permeability}

To investigate the temporal dynamics of membrane transport across the permeabilized membranes, fluorophores were added to the outer solution instead of the inner solution, detailed in (Caveney et al., 2019). Three fluorophores were used, fluorescein ( 332 Da), AF633 ( 1.2 $\mathrm{kDa})$, and AF647 conjugated to transferrin ( $\sim 80 \mathrm{kDa})$, to span the range of protein expression resources, amino acids ( $\sim 110 \mathrm{Da})$, nucleotides ( $\sim 650 \mathrm{Da})$, proteins ( $>20 \mathrm{kDa})$. Fluorescein crossed the membrane in all vesicles by the end of the experiment (SI Figure 2A). The vesicle in the black box is representative. AF633 had vesicles with three behaviors (SI Figure 2B) vesicles in boxes are representative). Behavior (1) vesicles began with high concentrations of AF633 and photobleached. Behavior (2) vesicles started with no AF633 and increased in fluorescence. Behavior (3) vesicles did not increase in fluorescence throughout the experiment. AF647 did not cross the membrane during the experiments (SI Figure $2 \mathrm{C}$ ). These results show all vesicles are permeable to small molecules but are impermeable to larger molecules.

On average, fluorescence of AF633 and AF647 in vesicles did not increase during the experiment, however fluorescein increased (SI Figure 2D). The fluorescein curve was fit by functions derived from different models of membrane permeabilization. The dashed line assumed first order reaction kinetics, the solid and dotted lines assume permeability increases with time either linearly or sigmoidally. The fluorescein data were better fit by models where permeability increases as the vesicles are exposed to more light.

\section{Model of Protein Expression in Permeabilized Vesicles}

Protein expression inside permeabilized vesicles was modeled with a Gillespie simulation (Gillespie 1977). The model had four populations required to make protein: genes, G, that could not cross the membrane; large resources, $Q$, that could not cross the membrane; small molecules, R, that could cross the membrane slowly; and very small molecules, $L$, that could rapidly cross the membrane (SI Figure $1 A-D$ ). For all simulations $G=10$. The initial resource populations, $Q, R_{\text {in }}, L_{\text {in }}, R_{\text {out }}$, and $L_{\text {out }}$, in the high concentration conditions were equal to 100. The initial resource populations, $Q, L_{\text {in }}, R_{\text {out }}$, and $L_{\text {out }}$ in the low concentration conditions were equal to 33. The initial $R_{\text {in }}$ populations in the low concentration conditions were equal to 0 to model a depletion of inner resources before much protein could be made. The outer solution concentrations, $R_{\text {out }}$ and $L_{\text {out }}$, were assumed to be constant (SI Figure 3D) because the outer solution volume is much larger than the inner solution volume. Each of the four resource conditions was run for 100 trajectories. Each trajectory was simulated for 180 minutes and sampled every three minutes just as in the experiments. 


\section{Acknowledgements}

This research was conducted at the Center for Nanophase Materials Sciences, which is a DOE Office of Science User Facility. P.M.C. and S.E.N. also acknowledge Graduate Fellowships from the Bredesen Center for Interdisciplinary Research and Graduate Education, University of Tennessee, Knoxville. The authors would like to thank Osaka University and Dr. Tetsuya Yomo for providing pEToppYB plasmid, and Dr. Maike Hansen and the Dr. Leor Weinberger laboratory for useful discussions.

\section{Author Contributions}

Conceptualization, P.M.C., C.P.C., and M.L.S.; Methodology, P.M.C., C.P.C., and M.L.S.; Software, P.M.C.; Formal Analysis, P.M.C., M.L.S.; Investigation, P.M.C., R.M.D.; Writing Original Draft, P.M.C., W.T.M., and M.L.S.; Writing - Reviewing and Editing, P.M.C. and M.L.S.; Visualization, P.M.C.; Supervision, C.P.C., M.L.S.; Funding Acquisition, M.L.S.

\section{Declaration of Interests}

The authors declare no competing interests. 


\section{$\underline{\text { References }}$}

Braga, R.M., Dourado, M.N., and Araújo, W.L. (2016). Microbial interactions: ecology in a molecular perspective. Braz. J. Microbiol. 47, 86-98.

Carrara, P., Altamura, E., D’Angelo, F., Mavelli, F., and Stano, P. (2018). Measurement and numerical modeling of cell-free protein synthesis: combinatorial block-variants of the PURE system. Data 3, 41.

Caveney, P.M., Norred, S.E., Chin, C.W., Boreyko, J.B., Razooky, B.S., Retterer, S.T., Collier, C.P., and Simpson, M.L. (2016). Resource Sharing Controls Gene Expression Bursting. ACS Synth. Biol.

Caveney, P.M., Dabbs, R.M., McClintic, W.T., Norred, S.E., Collier, C.P., and Simpson, M.L. (2019). Controlling Cell-Free Gene Expression Behavior by Tuning Membrane Transport Properties. BioRxiv 604454.

Chalmeau, J., Monina, N., Shin, J., Vieu, C., and Noireaux, V. (2011). a-Hemolysin pore formation into a supported phospholipid bilayer using cell-free expression. Biochim. Biophys. Acta BBA-Biomembr. 1808, 271-278.

Collier, C.P., and Simpson, M.L. (2011). Micro/nanofabricated environments for synthetic biology. Curr. Opin. Biotechnol. 22, 516-526.

Exterkate, M., Caforio, A., Stuart, M.C., and Driessen, A.J. (2017). Growing membranes in vitro by continuous phospholipid biosynthesis from free fatty acids. ACS Synth. Biol. 7, 153-165.

Flemming, H.-C., Wingender, J., Szewzyk, U., Steinberg, P., Rice, S.A., and Kjelleberg, S. (2016). Biofilms: an emergent form of bacterial life. Nat. Rev. Microbiol. 14, 563.

Garcia, D.C., Mohr, B.P., Dovgan, J.T., Hurst, G.B., Standaert, R.F., and Doktycz, M.J. (2018). Elucidating the potential of crude cell extracts for producing pyruvate from glucose. Synth. Biol. 3, ysy006.

Gillespie, D.T. (1977). Exact stochastic simulation of coupled chemical reactions. J. Phys. Chem. 81, 2340-2361.

Gogarten, J.P., and Townsend, J.P. (2005). Horizontal gene transfer, genome innovation and evolution. Nat. Rev. Microbiol. 3, 679.

Hansen, M.M., Desai, R.V., Simpson, M.L., and Weinberger, L.S. (2018a). Cytoplasmic amplification of transcriptional noise generates substantial cell-to-cell variability. Cell Syst. 7 , 384-397.

Hansen, M.M., Wen, W.Y., Ingerman, E., Razooky, B.S., Thompson, C.E., Dar, R.D., Chin, C.W., Simpson, M.L., and Weinberger, L.S. (2018b). A post-transcriptional feedback mechanism for noise suppression and fate stabilization. Cell 173, 1609-1621.

Karim, A.S., and Jewett, M.C. (2016). A cell-free framework for rapid biosynthetic pathway prototyping and enzyme discovery. Metab. Eng. 36, 116-126. 
Miller, M.B., and Bassler, B.L. (2001). Quorum sensing in bacteria. Annu. Rev. Microbiol. 55, 165-199.

Moore, S.J., MacDonald, J.T., Wienecke, S., Ishwarbhai, A., Tsipa, A., Aw, R., Kylilis, N., Bell, D.J., McClymont, D.W., and Jensen, K. (2018). Rapid acquisition and model-based analysis of cell-free transcription-translation reactions from nonmodel bacteria. Proc. Natl. Acad. Sci. 201715806.

Nishimura, K., Matsuura, T., Sunami, T., Fujii, S., Nishimura, K., Suzuki, H., and Yomo, T. (2014a). Identification of giant unilamellar vesicles with permeability to small charged molecules. RSC Adv. 4, 35224-35232.

Nishimura, K., Tsuru, S., Suzuki, H., and Yomo, T. (2014b). Stochasticity in gene expression in a cell-sized compartment. ACS Synth. Biol. 4, 566-576.

Noireaux, V., and Libchaber, A. (2004). A vesicle bioreactor as a step toward an artificial cell assembly. Proc. Natl. Acad. Sci. U. S. A. 101, 17669-17674.

Norred, S.E., Caveney, P.M., Chauhan, G., Collier, L.K., Collier, C.P., Abel, S.M., and Simpson, M.L. (2018). Macromolecular Crowding Induces Spatial Correlations That Control Gene Expression Bursting Patterns. ACS Synth. Biol.

Pardee, K., Green, A.A., Takahashi, M.K., Braff, D., Lambert, G., Lee, J.W., Ferrante, T., Ma, D., Donghia, N., and Fan, M. (2016). Rapid, low-cost detection of Zika virus using programmable biomolecular components. Cell 165, 1255-1266.

Pautot, S., Frisken, B.J., and Weitz, D.A. (2003). Production of unilamellar vesicles using an inverted emulsion. Langmuir 19, 2870-2879.

Perez, J.G., Stark, J.C., and Jewett, M.C. (2016). Cell-free synthetic biology: engineering beyond the cell. Cold Spring Harb. Perspect. Biol. 8, a023853.

Sakatani, Y., Ichihashi, N., Kazuta, Y., and Yomo, T. (2015). A transcription and translationcoupled DNA replication system using rolling-circle replication. Sci. Rep. 5, 10404.

Scott, A., Noga, M.J., de Graaf, P., Westerlaken, I., Yildirim, E., and Danelon, C. (2016). Cellfree phospholipid biosynthesis by gene-encoded enzymes reconstituted in liposomes. PloS One 11, e0163058.

Shimizu, Y., Inoue, A., Tomari, Y., Suzuki, T., Yokogawa, T., Nishikawa, K., and Ueda, T. (2001). Cell-free translation reconstituted with purified components. Nat. Biotechnol. 19, 751755.

Shin, J., and Noireaux, V. (2012). An E. coli cell-free expression toolbox: application to synthetic gene circuits and artificial cells. ACS Synth. Biol. 1, 29-41.

Siegal-Gaskins, D., Tuza, Z.A., Kim, J., Noireaux, V., and Murray, R.M. (2014). Gene circuit performance characterization and resource usage in a cell-free "breadboard." ACS Synth. Biol. 3, 416-425. 
Siuti, P., Retterer, S.T., and Doktycz, M.J. (2011). Continuous protein production in nanoporous, picolitre volume containers. Lab. Chip 11, 3523-3529.

Sun, Z.Z., Hayes, C.A., Shin, J., Caschera, F., Murray, R.M., and Noireaux, V. (2013). Protocols for implementing an Escherichia coli based TX-TL cell-free expression system for synthetic biology. J. Vis. Exp. JoVE.

Tinevez, J.-Y., Perry, N., Schindelin, J., Hoopes, G.M., Reynolds, G.D., Laplantine, E., Bednarek, S.Y., Shorte, S.L., and Eliceiri, K.W. (2017). TrackMate: An open and extensible platform for single-particle tracking. Methods 115, 80-90.

Trifonov, E.N. (2011). Vocabulary of definitions of life suggests a definition. J. Biomol. Struct. Dyn. 29, 259-266.

Van Haastert, P.J., and Devreotes, P.N. (2004). Chemotaxis: signalling the way forward. Nat. Rev. Mol. Cell Biol. 5, 626.

Yoshiyama, T., Ichii, T., Yomo, T., and Ichihashi, N. (2018). Automated in vitro evolution of a translation-coupled RNA replication system in a droplet flow reactor. Sci. Rep. 8, 11867. 


\section{A}

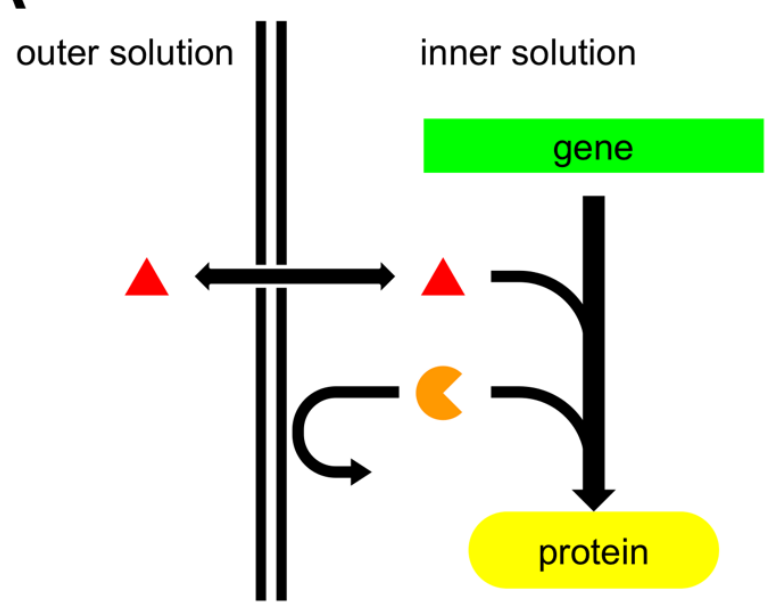

B

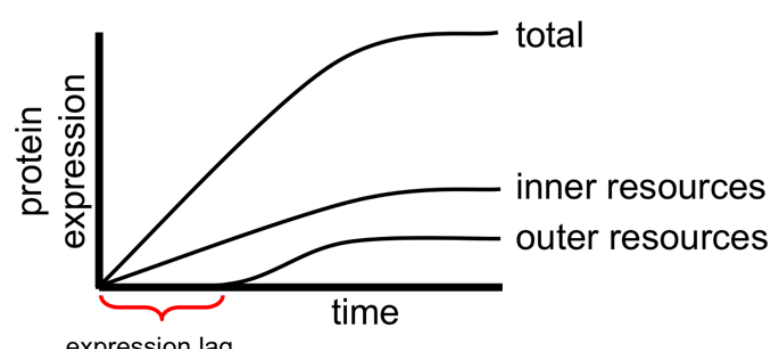

expression lag

Figure 1. Resource location (internal or external) affects gene expression behavior. (A) Gene expression is affected by the encapsulated (orange circular sector) and external (red triangle) molecular resources. (B) The gene expression transient is the sum of two components: one controlled by the internal resource concentration and one controlled by external resource concentration. The expression transient due to external resources should experience a delay (labeled here as expression lag) related to the membrane transport properties. 


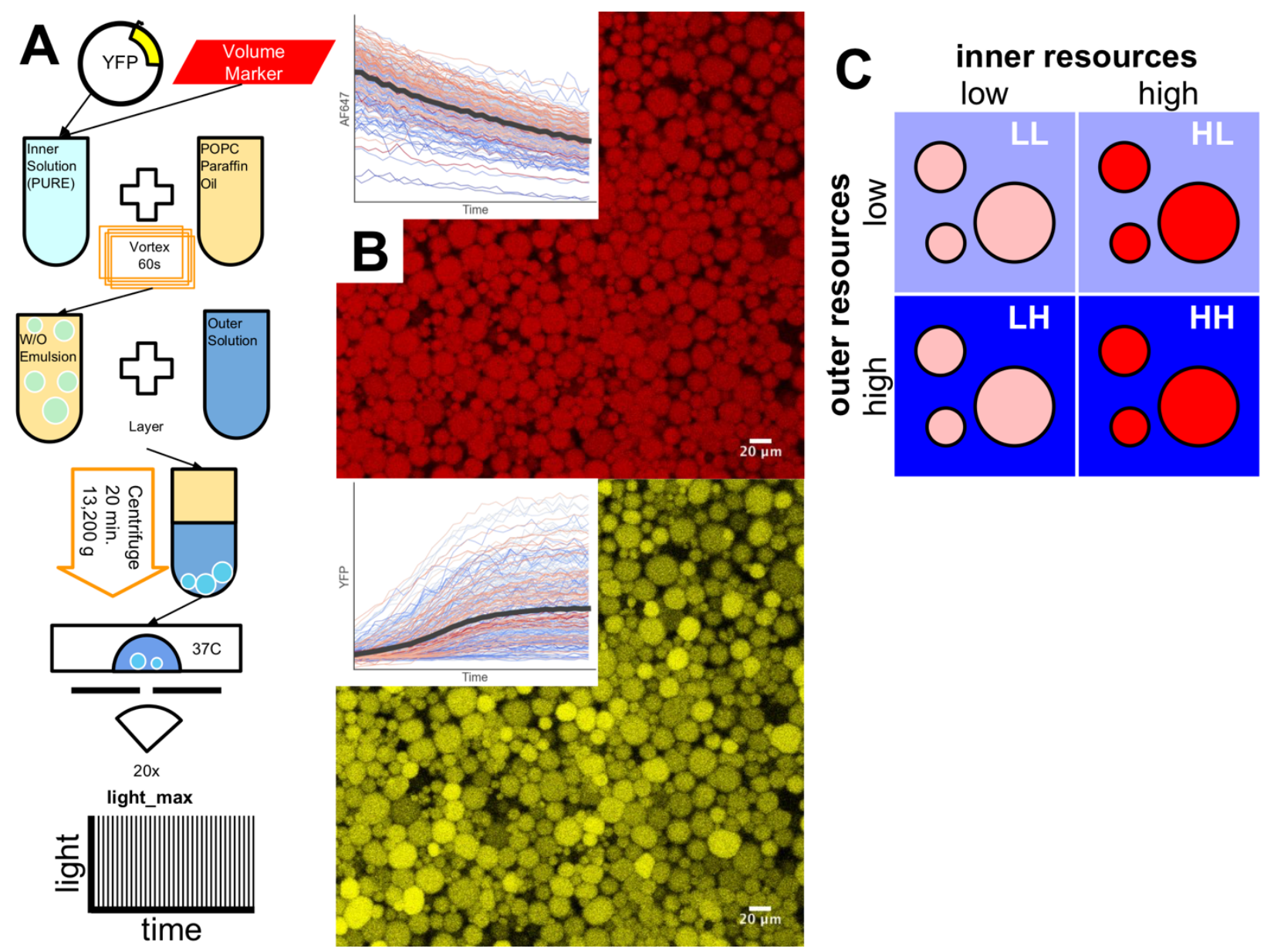

Figure 2. Gene expression in permeable lipid membranes with differing location of expression resources. (A) Method for making and imaging vesicles. Vesicles were imaged using a protocol, light_max, to cause membrane permeability (Caveney et al., 2019). (B) Representative images of the AF647 volume marker and YFP fluorescence in vesicles. Insets show transient behavior of AF647 (photobleaching decay) and YFP fluorescence. (C) Varying the location of essential gene expression resources by changing inner and outer solution concentrations. 

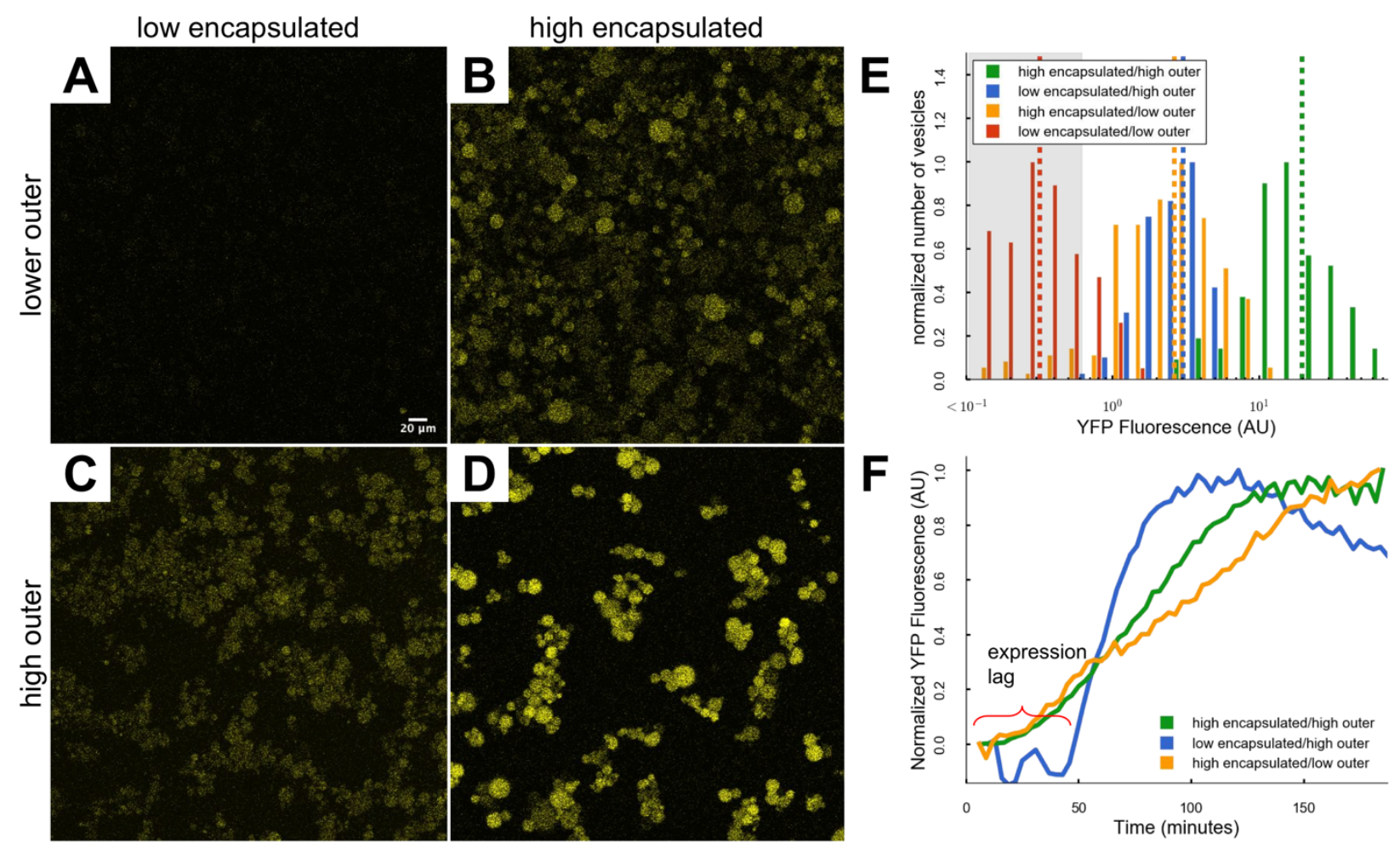

Figure 3. Expression resource location controls gene expression level and dynamics. (A) The $t=2$ hour image of YFP expressed with the low encapsulated/low outer concentration condition. (B) The $t=2$ hour image of YFP expressed with the high encapsulated/low outer concentration condition. (C) The $t=2$ hour image of YFP expressed under the low encapsulated/high outer concentration condition. (D) The $t=2$ hour image of YFP expressed under the high encapsulated/high outer condition. (E) Distributions of YFP produced under each of the four resource concentration conditions. The dashed vertical lines indicate the means of each distributions. Gray shaded region denotes vesicles with YFP intensities indistinguishable from the background. $(F)$ Average transient behavior of vesicles under the three conditions that produced significant amounts of protein. 


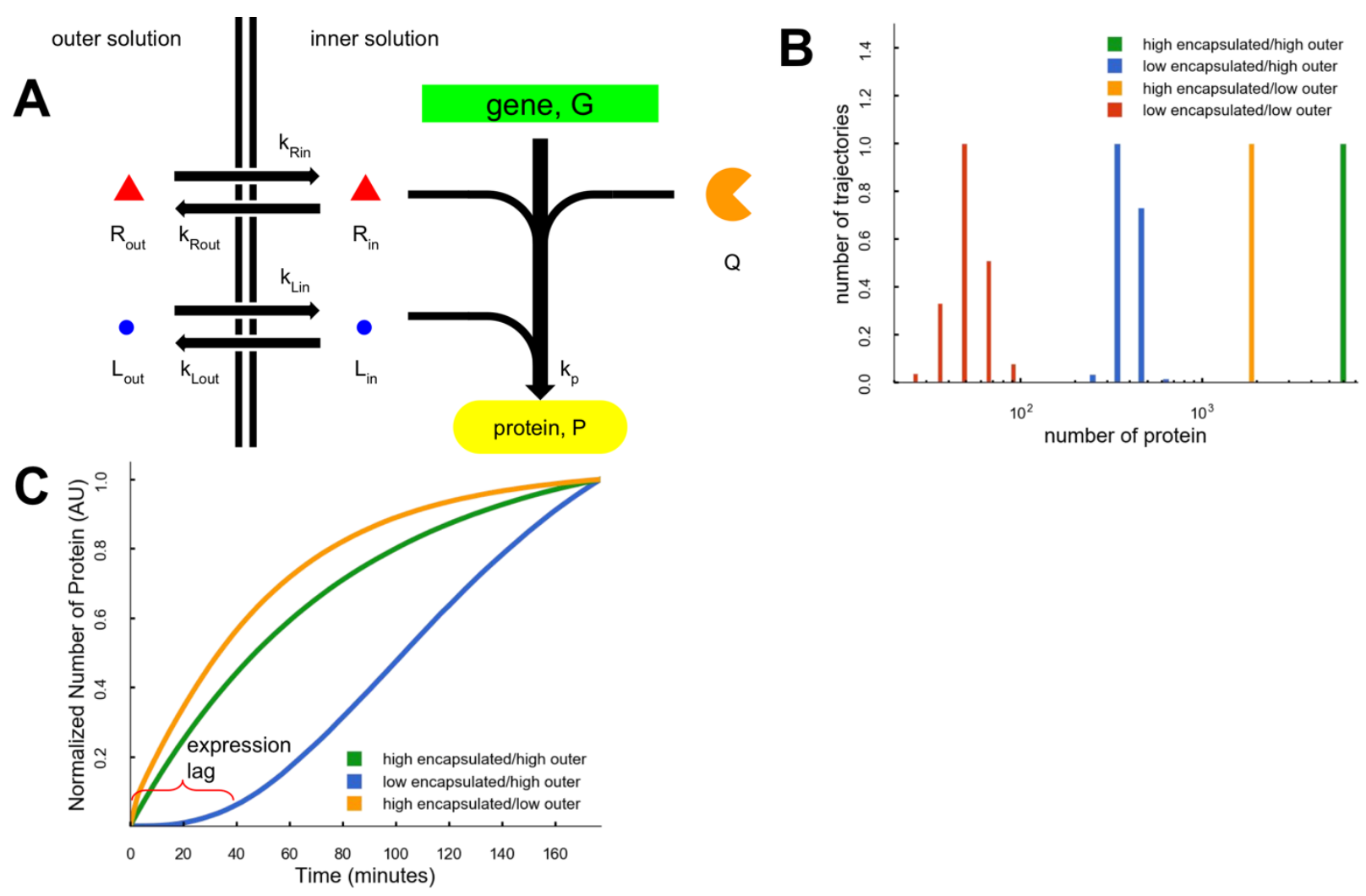

Figure 4. Model of protein expression with resources divided by a permeable membrane. (A) Graphic of the Gillespie simulation. Species $G$ and $Q$ do not cross the membrane while species $R$ and $L$ cross with different rates, $k_{R}$ and $k_{L}$. (B) Distributions of final protein for each of the four conditions. (C) Transients of protein expression for the three conditions that make significant amounts of protein. 